\title{
Advances in Nonlinear Optical Materials and Devices
}

Robert L. Byer

Department of Applied Physics, Edward L. Ginzton Laboratory Stanford University, Stanford, CA 94305

\section{Abstract}

The recent progress in the application of nonlinear techniques to extend the frequency of laser sources has come from the joint progress in laser sources and in nonlinear materials. A brief summary of the progress in diode pumped solid state lasers is followed by an overview of progress in nonlinear frequency extension by harmonic generation and parametric processes. Improved nonlinear materials including bulk crystals, quasiphasematched interactions, guided wave devices and quantum well intersubband studies is discussed with the idea of identifying areas of future progress in nonlinear materials and devices.

Keywords: nonlinear materials, second harmonic generation, parametric oscillation, $\mathrm{MgO}: \mathrm{LiNbO}_{3}, \mathrm{AgGaSe}_{2}, \mathrm{BaB}_{2} \mathrm{O}_{4}, \mathrm{LiB}_{3} \mathrm{O}_{5}$, Quasiphasematching, guided wave doubling, AlGaAs quantum well nonlinearity

\section{INTRODUCTION}

The introduction of the modern high power diode laser array by $D$. R. Scifres, R. D. Burnham and W. Streifer in 1978 [1] opened the door to rapid advances in diode pumped solid state laser sources. That progress is the subject of reviews $[2,3]$. The progress in the spatial and temporal coherence of the solid state laser sources coupled with the advances in nonlinear materials has led to an explosion of activity in nonlinear device studies and demonstrations. Now, thirty years after the first demonstration of frequency conversion from the red to the blue by Franken and his group of students at the University of Michigan, nonlinear optical devices are on the threshold of making the transition from the laboratory into widespread applications. Lasers are now common in homes. It is but a matter of time before nonlinear optical devices will also be found in homes and perhaps automobiles.

(NASA-CR-192681) ADVANCES IN

N9 3-25281

NONLINEAR OPTICAL MATERIALS ANO

DEVICES (Stanford Univ.) $14 \mathrm{P}$
Unclas 


\section{DIODE PUMPED SOLID STATE LASERS}

The use of a GaAs diode to pump a solid state laser was suggested in 1963 by Newman [4] who anticipated the advantages of small size and efficiency that diode pumping offered. What was probably not anticipated was the significant improvement in coherence gained by diode pumping a solid state laser medium. The first monolithic diode pumped Nd:YAG laser operated at a linewidth of less than $10 \mathrm{kHz}$ [5]. Since that first demonstration the linewidth of diode pumped Nd:YAG oscillators has improved rapidly.

\subsection{Improved Coherence - The Nonplanar Ring Oscillator}

The need for a highly stable, single frequency laser oscillator for coherent radar measurement of wind velocity led Kane and Byer to invent the nonplanar ring oscillator in 1984 [6]. The comprehensive theory of the nonplanar ring oscillator was developed using optical equivalence theorems by Nilsson et. al.[7]. Measurements of the relative linewidths of successive monolithic nonplanar ring laser oscillators over a period of two years led to beatnote linewidths of $500 \mathrm{~Hz}, 30 \mathrm{~Hz}, 3 \mathrm{~Hz}$ [8] and finally to subhertz levels [9].

The frequency fluctuations and coherence properties of the monolithic nonplanar ring oscillators have been studied extensively $[10,11$,$] . The$ oscillators are found to be shot noise limited above a frequency of $100 \mathrm{kHz}$. The frequency noise can be modeled by the thermal response of the Nd:YAG crystal to the amplitude fluctuations of the diode laser pump source. Control of the diode pump laser amplitude leads to a reduction in the $1 / \mathrm{f}$ frequency noise spectrum of the nonplanar ring oscillator at low frequencies.

\subsection{Injection Locking}

The power level of nonplanar ring oscillators has steadily improved with the availability of higher power diode laser pump sources. In 1986 the available power was $3 \mathrm{~mW}$. By 1989 the power had risen to $40 \mathrm{~mW}$ and by 1991 to $300 \mathrm{~mW}$. There is reason to believe that the output power levels of these sub-kilohertz oscillators will exceed the $1 \mathrm{~W}$ level but be limited to less than the $100 \mathrm{~W}$ level. However, the high coherence of the nonplanar ring oscillator can be preserved and the power level increased by using injection locking. Following the approach first suggested by Robert Adler in 1946 [12], Nabors et. al. [13] demonstrated that a $40 \mathrm{~mW}$ nonplanar ring master oscillator could control the spectral characteristics of a $13 \mathrm{~W} \mathrm{cw}$ lamp pumped Nd:YAG ring slave oscillator by injection locking. The power has since be increased to $18 \mathrm{~W}$. This single frequency, single transverse mode high power Nd:YAG laser source has been an important element in the progress in nonlinear optical devices discussed below. 


\subsection{High Average Power Diode Pumped Solid State Lasers}

The continued improvement in the power and efficiency of diode laser arrays for pumping solid state lasers has led to increased output power. The early work by Sipes [14], in which he demonstrated the use of a diode laser array to end pump a Nd:YAG laser, has been extended by others to powers exceeding the multiwatt level $[15,16]$. Further improvements in average power is expected with the use of side pumping and the slab geometry[17] for controlling the optical distortions caused by thermal loading of the laser medium. The average power scaling limits for diode pumped solid state lasers has been explored by Basu and Byer who predict average power levels in excess of $20 \mathrm{~kW}$ [18].

The experimental progress toward high average power diode pumped lasers is proceeding in a number of laboratories. At Stanford we have designed a side pumped, $10 \mathrm{~W} \mathrm{cw}$ TEMoo mode Nd:YAG laser that will be pumped by sixty one-Watt fiber coupled diode lasers. Also under construction is a $100 \mathrm{~W}$, $\mathrm{cw}$ slab geometry Nd:YAG laser to be pumped by fifty fiber coupled $10 \mathrm{~W}$ diode laser bars. The Nd:YAG laser gain medium for these laser oscillators is shaped in a slab geometry with a length of $24 \mathrm{~mm}$ and a thickness of $1.5 \mathrm{~mm}$. When available, these injection locked, single frequency, diffraction limited laser sources will offer both opportunities and challenges to nonlinear optical frequency conversion.

\section{NONLINEAR FREQUENCY EXTENSION}

High conversion efficiency of laser radiation by nonlinear optical techniques requires adequate laser power. For low power, continuous wave, laser sources, placing the nonlinear crystal inside to the laser cavity or in an external resonant cavity allows resonant enhancement of the laser power for efficient second harmonic generation (SHG). Similarly, to achieve tunable coherent output through optical parametric oscillation (OPO) requires adequate pump laser power or low loss in the nonlinear crystal since the OPO threshold varies as the square of the optical losses. Progress in SHG and in OPO operation is summarized briefly in the next two sections.

\subsection{Internal and External Resonant Second Harmonic Generation}

The early work in SHG took advantage of the enhanced field within the laser cavity. Fan et. al. [19] used $\mathrm{MgO}_{\mathrm{LiNbO}}$ to double a diode pumped Nd:YLF laser pumped by a $30 \mathrm{~mW}$ diode laser. That first experiment yielded $0.3 \mathrm{~mW}$ of output at the green second harmonic. Baer [20] demonstrated efficient second harmonic output using KTP as the nonlinear crystal internal to a diode pumped Nd:YLF laser and recognized an amplitude stability problem due to the nonlinear coupling of the laser axial modes. That work was extended recently to higher powers by Oka and Kubota [21]. Internal 
second harmonic generation has the advantage of being relative easy to implement but the disadvantages of introducing loss elements into the laser resonator and coupling the laser modes through the nonlinear interaction.

External resonant SHG was first proposed in 1966 by Ashkin et. al. [22]. The implementation of external resonant SHG at high efficiency awaited the availability of the single frequency nonplanar ring oscillator and low loss, high optical quality, nonlinear crystals. Using a nonplanar ring oscillator with $53 \mathrm{~mW}$ of $\mathrm{cw}$ output power, Kozlovsky et. al. [23] generated $30 \mathrm{~mW}$ of $\mathrm{cw}$ green in a monolithic external resonant cavity formed of $\mathrm{MgO}: \mathrm{LiNbO}_{3}$. The $56 \%$ conversion efficiency from $1064 \mathrm{~nm}$ to $532 \mathrm{~nm}$ in a $12.5 \mathrm{~mm}$ long ring resonator of $\mathrm{MgO}: \mathrm{LiNbO}_{3}$ established a record in 1988 which has only recently been exceeded with $200 \mathrm{~mW}$ of green produced at $65 \%$ efficiency [24].

External resonant second harmonic generation was extended in 1990 to the efficient doubling of a diode laser source by Kozlovsky et. al. [25] Using a monolithic ring resonator of $\mathrm{KNbO}_{3}$, Kozlovsky et. al. demonstrated $41 \mathrm{~mW}$ of blue output from $105 \mathrm{~mW}$ of input diode laser power. The electrical efficiency of this blue source was $10 \%$.

Recently, external resonant SHG was extended to high average power levels using the $18 \mathrm{~W}$ injection locked Nd:YAG laser described in Figure 1. An antireflection coated, $6 \mathrm{~mm}$ long, $\mathrm{LiB}_{3} \mathrm{O}_{5}(\mathrm{LBO})$ crystal placed within the external resonant cavity generated more than $6.5 \mathrm{~W}$ of $\mathrm{cW}$, single axial mode harmonic output at $532 \mathrm{~nm}$ from $18 \mathrm{~W}$ of incident $1064 \mathrm{~nm}$. The generated green output was diffraction limited and had a linewidth of $15 \mathrm{kHz}$.

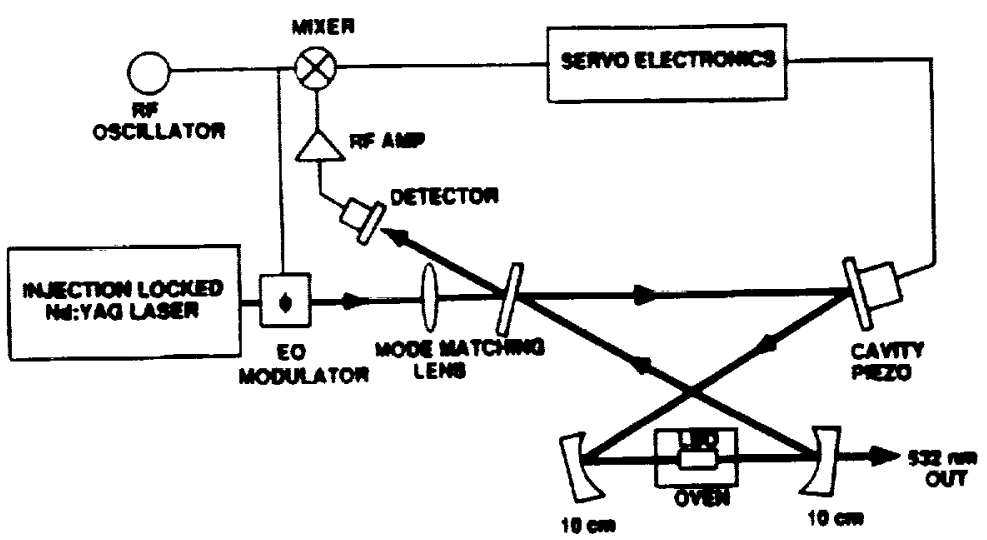

Figure 1. Schematic of external resonant second harmonic generation using an $18 \mathrm{~W}$ injection locked Nd:YAG source. $6.5 \mathrm{~W}$ of green was generated in a $6 \mathrm{~mm}$ long crystal of LBO at $36 \%$ conversion efficiency. The green output was diffraction limited with a linewidth of $15 \mathrm{kHz}$. 
In resonantly enhanced SHG the important figure of merit for the nonlinear crystal is the ratio of the nonlinear optical coefficient to the round trip loss; $d_{e f f} \alpha$. Thus it is as important to reduce the loss as it is to increase the nonlinearity. The impact of loss on conversion efficiency is illustrated in Figure 2 where the theoretical SHG conversion efficiency is shown vs round trip resonator loss. A loss of $2 \%$ limits the conversion efficiency to $40 \%$ while a loss of $0.2 \%$ raises the efficiency to $90 \%$.

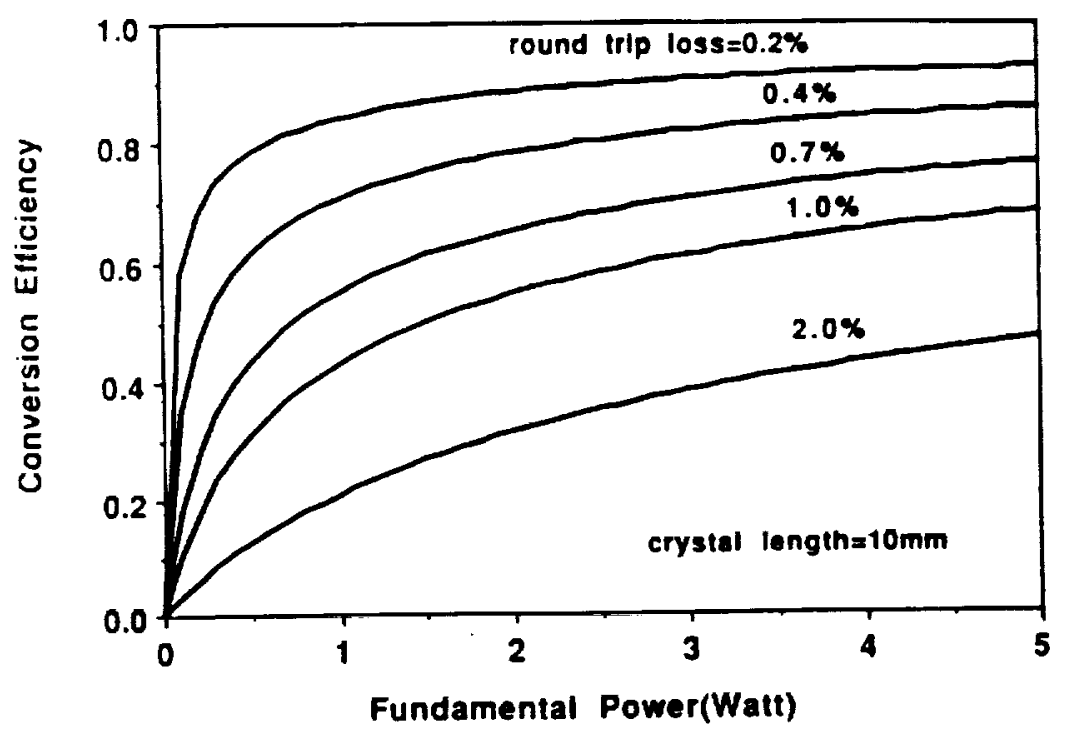

Figure 2. The theoretical conversion efficiency for $532 \mathrm{~nm}$ to $266 \mathrm{~nm}$ conversion by SHG in a BBO crystal by external resonant SHG. The parameter is the round trip resonator loss which includes the loss due to the nonlinear crystal.

The external resonant SHG apparatus shown in Figure 1 was also used to test the quality of "a new flavor" of lithium niobate; lithium rich lithium niobate fabricated by vapor transport [27]. Doubling efficiencies of greater than $69 \%$ were obtained at the $240 \mathrm{C}$ noncritical phasematching temperature with output power levels of up to $1.4 \mathrm{~W}$ [28]. The measured optical loss coefficient of $0.42 \% / \mathrm{cm}$ at $1064 \mathrm{~nm}$ for this material was adequate for high conversion efficiency but was not adequate to avoid thermal effects due to absorption at the resonant fundamental wave. The absorbed power at the fundamental led to hysterisis effects in the generation of the harmonic at the input power of $4.2 \mathrm{~W}$ at the fundamental. The onset of thermal effects is another reason to reduce the optical loss of the nonlinear crystal especially for high average power applications. 


\subsection{Optical Parametric Oscillators}

In 1965 Giordmaine and Miller [29] demonstrated the first optical parametric oscillator in $\mathrm{LiNbO}_{3}$. The early devices were not stable due to the poor frequency stability of the laser pump sources. The availability of the nonplanar ring oscillator followed by resonant SHG in monolithic $\mathrm{LiNbO}_{3}$ provided a $532 \mathrm{~nm}$ single frequency pump source and opened the possibility of $\mathrm{cw}$ optical parametric oscillation. Nabors et.al. [30] achieved cw OPO operation in a monolithic ring resonator fabricated using $\mathrm{MgO}: \mathrm{LiNbO}_{3}$. The threshold was $10 \mathrm{~mW}$. At twice threshold the OPO operated at a conversion efficiency from green to tunable infrared output of 34\%. Furthermore, The OPO operated in a single frequency with a linewidth that reproduced the $10 \mathrm{kHz}$ linewidth of the pump laser source. The OPO was electric field tuned over a total range of $10 \mathrm{THz}$ by the application of an electric field of $924 \mathrm{~V}$. The coherence properties of this cw, monolithic OPO were studied by Nabors et. al. [31]. It was discovered that at degeneracy, where the two output frequencies are at the subharmonic of the pump frequency, the OPO subharmonic output phaselocked to the pump phase to form an optical frequency divider. The frequency tuning characteristics of the $\mathrm{cw}$ monolithic $\mathrm{LiNbO}_{3} \mathrm{OPO}$ have been studied theoretically in detail [32]. It appears that by tuning two variables simultaneously, the voltage on the crystal and the pump frequency, for example, the doubly resonant OPO can be continuously tuned over $10 \mathrm{GHz}$ without axial mode jumps [32]. The cw OPO offers the potential for widely tunable output that preserves the coherence of the pump laser source.

\section{IMPROVED NONLINEAR MATERIALS}

The development of a nonlinear material is an investment that takes more than one decade. The material must, of course, be nonlinear. However, it must also satisfy a number of additional optical and mechanical parameters to be useful in devices. These parameters include: high optical quality and transparency in the region of interest; the ability to prepare or grow the material; the ability to optically polish or optically coat the surfaces; good mechanical strength; and finally the ability to phasematch the nonlinear interaction. It is the latter constraint that places the most severe restriction on the availability of nonlinear crystals for device applications.

The nonlinear coefficients of a number of widely used nonlinear crystals has been remeasured recently using second harmonic generation by Eckardt et. al. [33]. The nonlinear coefficient values obtained for KDP agreed with previous measurements but values for $\mathrm{BBO}_{1} \mathrm{LiIO}_{3}, \mathrm{MgO}_{\mathrm{g}} \mathrm{LiNbO}_{3}$ and KTP were found to be in need of substantial revision. The downward revision of the nonlinear coefficient values were found to be in agreement with recent crystal performance measurements in nonlinear device applications. 


\subsection{Bulk Nonlinear Crystals}

$\mathrm{LiNbO}_{3}$ was first grown and applied to nonlinear optical interactions in 1965. The crystal has undergone significant improvements over the past twenty five years. However, $\mathrm{LiNbO}_{3}$ still is subject to improvements in both growth and in optical properties. For example, the discovery that magnesium doping of litium niobate raises the phasematching temperature was made in the late $1960 \mathrm{~s}$. It was discovered only very recently that for a precise magnesium doping near $5 \%$, the $\mathrm{MgO}: \mathrm{LiNbO}_{3}$ crystal does not exhibit a photorefractive response even at room temperature [34]. This discovery may open the door for wider applications of $\mathrm{MgO}: \mathrm{LiNbO}_{3}$ to devices for both harmonic generation and for optical parametric oscillation.

The chalcopyrite crystals were first grown for nonlinear optical applications in 1972 . Only recently have the crystals $\mathrm{AgGaS}_{2}$ and $\mathrm{AgGaSe}_{2}$ become available on a commercial basis. These crystals phasematch for infrared generation by mixing and by parametric oscillation across the near to mid-infrared [35]. Improvements are still needed to reduce the optical losses and to stabilize the surface against degradation from high power optical radiation.

Recent additions to the collection of useful bulk crystals is $\mathrm{BaB}_{2} \mathrm{O}_{4}(\mathrm{BBO})$ and $\mathrm{LiB}_{3} \mathrm{O}_{5}$ (LBO). The crystal $\mathrm{BBO}$ has proved useful for high peak power harmonic generation of the Nd:YAG laser source and for use as a high peak power pulsed OPO [36]. The crystal still needs reduction in optical scatter and in surface preparation to minimize loss. LBO has proven useful for SHG of a cw Nd:YAG because it noncritically phasematches at $150 \mathrm{C}$ for doubling $1064 \mathrm{~nm}$. However, LBO is early in its crystal growth development and suffers from optical scatter due to inclusions. LBO has been used recently for pulsed OPO operation [37].

\subsection{Quasiphasematching}

Quasiphasematching (QPM) was the first technique proposed to correct for crystal dispersion to achieve phase velocity matching between the fundamental and the harmonic wave [38]. In this technique, for which a patent was awarded to $\mathrm{N}$. Bloembergen in 1968 [39], the nonlinear coefficient sign is modulated spatially to offset the phasemismatch caused by material dispersion. The demonstration of quasiphasematched interactions was achieved with limited success until recently with the growth of QPM crystals of $\mathrm{LiNbO}_{3}$ in both the bulk form [40] and with the preparation of periodic poled lithium niobate (PPLN) in the form of a waveguided structure [41]. 


\section{Bulk Crystal QPM}

The successful growth of PPLN in bulk form was achieved at Stanford using a Laser Heated Pedestal Growth Apparatus [42]. Crystal fibers of aaxis orientation were grown with a domain reversal distance of $3.4 \mu \mathrm{m}$ for first order doubling of the $1064 \mathrm{~nm}$ line of Nd:YAG. The a-axis oriented crystal allowed the use of the largest nonlinear coefficient in $\mathrm{LiNbO}_{3}$ such that $\mathrm{d}_{\text {eff }}=2 \mathrm{~d}_{33} / \pi=20.9 \mathrm{pm} / \mathrm{V}$. The observed second harmonic conversion efficiency increased quadradically with the fiber length up to a distance of $680 \mu \mathrm{m}$ or 280 domains. When the $1.24 \mathrm{~mm}$ long sample was placed in the external resonant cavity shown in Figure 1, up to $1.7 \mathrm{~W}$ of $\mathrm{cw}$ green was generated for $4.2 \mathrm{~W}$ of incident $1064 \mathrm{~nm}$ [43]. Figure 3 shows the generated second harmonic power vs the incident fundamental power. The solid line is the theoretical fit assuming that the effective phasematched crystal length is $0.56 \mathrm{~mm}$. The conversion efficiency, defined as the incident power mode matched into the external resonator divided by the generated green at the exit face of the periodically poled lithium niobate crystal reached $42 \%$ at $2 \mathrm{~W}$ of incident power.

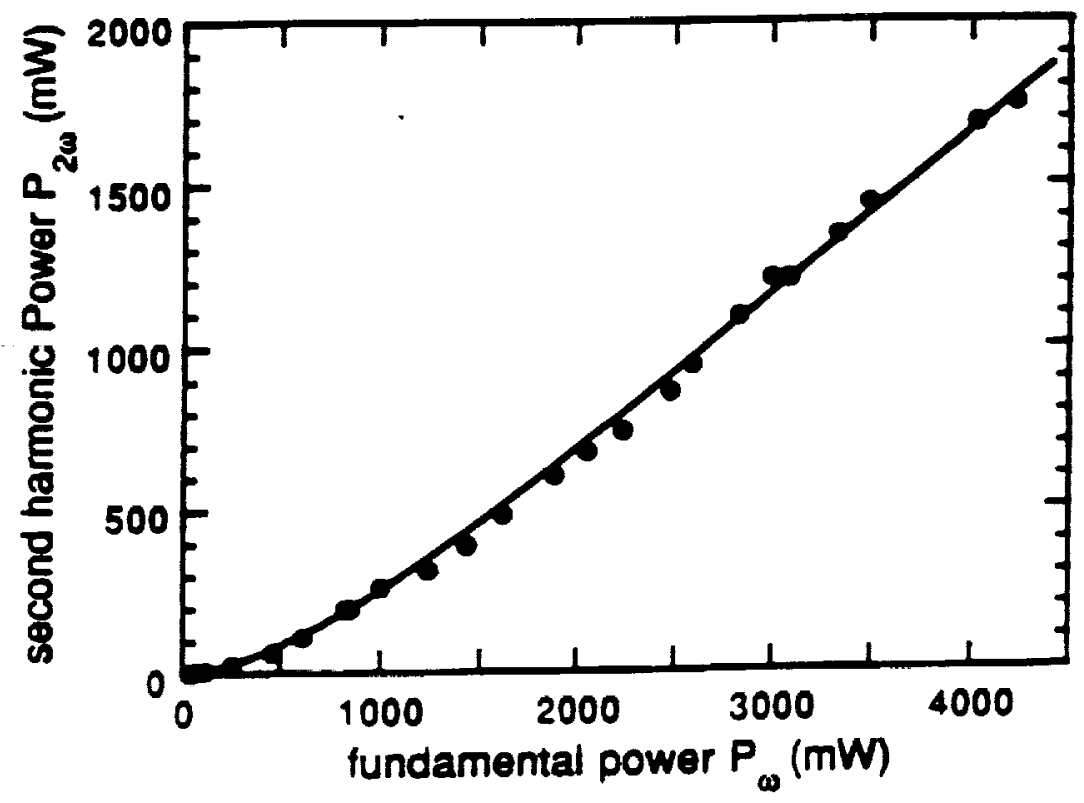

Figure 3. Second harmonic generation using quasiphasematching in melt grown periodically poled $\mathrm{LiNbO}_{3}$ (PPLN). The external resonant cavity shown in Figure 1 was used to generate $1.4 \mathrm{~W}$ of $\mathrm{cw} 532 \mathrm{~nm}$ for $4.2 \mathrm{~W}$ of incident $1064 \mathrm{~nm}$. The dots are experimental data. The solid line is the theoretical fit for an effective interaction length of $0.56 \mathrm{~mm}$. 
The demonstration of efficient device performance in PPLN illustrates that the material has high optical quality, large nonlinear coefficient and low loss. Further, PPLN crystals do not show signs of photorefractive response even at room temperature under intense illumination with blue radiation. The advantages of quasiphasematching are clearly evident in this device demonstration. Further demonstration of QPM has been achieved by the generation of red, green and blue radiation using PPLN crystals with properly grown periods. The growth of crystals with particular phasematching characteristics can be extended to the generation of an arbitrary phasematching response by controlling the length and the phase of the domain reversals. The theory of QPM sensitivity to various imperfections in the domain reversals has been developed. The theory has been extended to the synthesis of the phase-matching response function by inverse Fourier transform synthesis [44].

\section{Guided Wave QPM}

QPM in a planar waveguide lithium niobate crystal was experimentally demonstrated by Lim et. al. [41]. The periodic domain structure was achieved using lithographically patterned titanium indiffusion at 1000 to $1100 \mathrm{C}$ on a lithium niobate wafer. The domains varied in length from 5 to $50 \mu \mathrm{m}$. A planar annealed proton-exchange waveguide resulted in a single TM mode planar guide at $1064 \mathrm{~nm}$. The SHG of $1064 \mathrm{~nm}$ took place in the planar guide over the $1 \mathrm{~mm}$ length of the domain that quasiphasematched. The observed SHG was approximately 1500 times larger than if the interaction had not been phasematched.

The use of a planar guide improves the conversion efficiency of the harmonic process by the ratio of $[L \Omega]^{1 / 2}$ relative to a focussed bulk interaction. The use of a channel guide improves the conversion efficiency by the ratio of $L \lambda$ compared to the bulk focussed case. For $L=1 \mathrm{~cm}$ planar and channel guides, the improvement factors are 100 and 10000 for SHG of $1000 \mathrm{~nm}$ radiation. Thus there is a significant gain for realizing QPM in a channel waveguide geometry.

Blue light at $410 \mathrm{~nm}$ was first demonstrated by Lim et. al. [45] in a channel guide of QPM lithium niobate. The normalized conversion efficiency was measured to be $37 \% / \mathrm{Wcm}^{2}$. The theoretically calculated normalized conversion efficiency assuming perfect spatial mode overlap is $350 \% / \mathrm{Wcm}^{2}$. For a $1 \mathrm{~cm}$ long channel guide, QPM lithium niobate should convert $50 \mathrm{~mW}$ of input to $1 \mathrm{~mW}$ of blue harmonic output. To date the best experimental observation is $1.5 \mathrm{~mW}$ of blue output for $200 \mathrm{~mW}$ of input.

The generation of blue light by guided wave QPM interaction is of commercial significance. Thus there has been rapid development of QPM in 
materials other than lithium niobate and there has been the development of techniques beyond chemical diffusion [46] to achieve period domain reversal. For example, czochralski grown periodic poled lithium niobate crystals using a $0.5 \%$ yttrium doped melt were prepared by Lu et. al. [47]. They demonstrated the generation of $430 \mathrm{~nm}$ and $435 \mathrm{~nm}$ light by doubling in structures that were 460 domains in length. Recently Mizuuchi et. al. [48] achieve $12 \mathrm{~mW}$ of blue light at $424 \mathrm{~nm}$ for $190 \mathrm{~mW}$ of incident power from a $\mathrm{Ti}: \mathrm{Al}_{2} \mathrm{O}_{3}$ laser source in QPM lithium tantalate. The third order QPM domains were $11.1 \mu \mathrm{m}$ in length and the interaction was in a channel guide. Bierlein et. al. [49] have extended periodic poling to KTP using barium diffusion to create a spatially segmented waveguide. They have demonstrated $25 \mathrm{~mW}$ of blue output for $200 \mathrm{~mW}$ of incident fundamental radiation. Finally, H. Ito has demonstrated periodic domain fabrication using electron beam writing in both $\mathrm{LiNbO}_{3}$ and $\mathrm{LiTaO}_{3}$ crystals [50]. Domain periods to $3.2 \mu \mathrm{m}$ have been written with domain reversals penetrating through a $1 \mathrm{~mm}$ thick sample.

In an interesting twist to $\mathrm{QPM}, \mathrm{Lim}$ et. al. [51] demonstrated $2 \mu \mathrm{m}$ infrared generation by mixing a $1.32 \mu \mathrm{m}$ Nd:YAG laser with the output from a $\mathrm{Ti}: \mathrm{Al}_{2} \mathrm{O}_{3}$ laser in $\mathrm{QPM} \mathrm{LiNbO}$. This difference frequency generation may find wide application as an inexpensive means to obtain tunable infrared radiation for chemical or gas monitoring or for process control applications.

The availability of large wafers of $\mathrm{LiNbO}_{3}$ and $\mathrm{LiTaO}_{3}$ and the improvements in methods of periodic poling these crystals to achieve QPM should allow rapid progress toward miniature blue sources by SHG of diode lasers. Methods of coupling the diode laser source into the waveguide and of optimizing the channel guide [52] must be achieved before the technology is capable of widespread use [53].

\subsection{Quantum Well Intersubband Nonlinearities}

The observation by West and Eglash [54] of intersubband absorption and the large oscillator strengths opened the possibility of extremely large second order nonlinear susceptibilities in quantum well structures. The predicted nonlinear response was experimentally measured for the first time by Fejer et. al. in 1989 [55]. The electric field biased nonlinearity in $92 \mathrm{~A}$ GaAs wells was measured to be $28,000 \mathrm{pm} / \mathrm{V}$. The magnitude and the sign of the nonlinearity could be changed by changing the sign of the bias electric field thus allowing for the possibility of QPM. Although this nonlinear response is extremely large compared to visibly transparent oxides or even to organic crystals, it is still well below the response that could be expected from a compositionally controlled asymmetric quantum well system. 
In recent work, Yoo et. al.[56] have designed and grown a compositionally asymmetric quantum well structure in GaAs. Figure 4a shows the structure of the asymmetric well and the calculated wavefunctions. The measured nonlinear susceptibility for this sample is shown in Figure $4 \mathrm{~b}$. The measurements were made by SHG of a $\mathrm{CO}_{2}$ laser. The solid line is the predicted nonlinear susceptibility and the diamonds are the experimental data. The nonlinear susceptibility reached a value of $58,000 \mathrm{pm} / \mathrm{V}$ or 320 times the bulk nonlinearity of GaAs. Of significance for device applications is the suppression of this nonlinear response to zero with proton bombardment. QPM devices in the infrared appear possible in quantum well GaAs by the use of proton bombardment to write the spatially modulated structures required to achieve phasematched interactions.

The ability to grow asymmetric quantum well structures with quasiphasematched interactions opens whole new avenues to efficient nonlinear interactions in the infrared. The extremely large nonlinear response allows the possibility of parametric amplifiers and efficient mixers. The ability to extend the quantum well structures to II-VI compounds allows near infrared or even visible devices to be contemplated. Finally, the combination of the large nonlinear susceptibility with low loss resonant structures on the semiconductor substrate allows for resonant surface emitters that eliminate the need for phasematching [57].
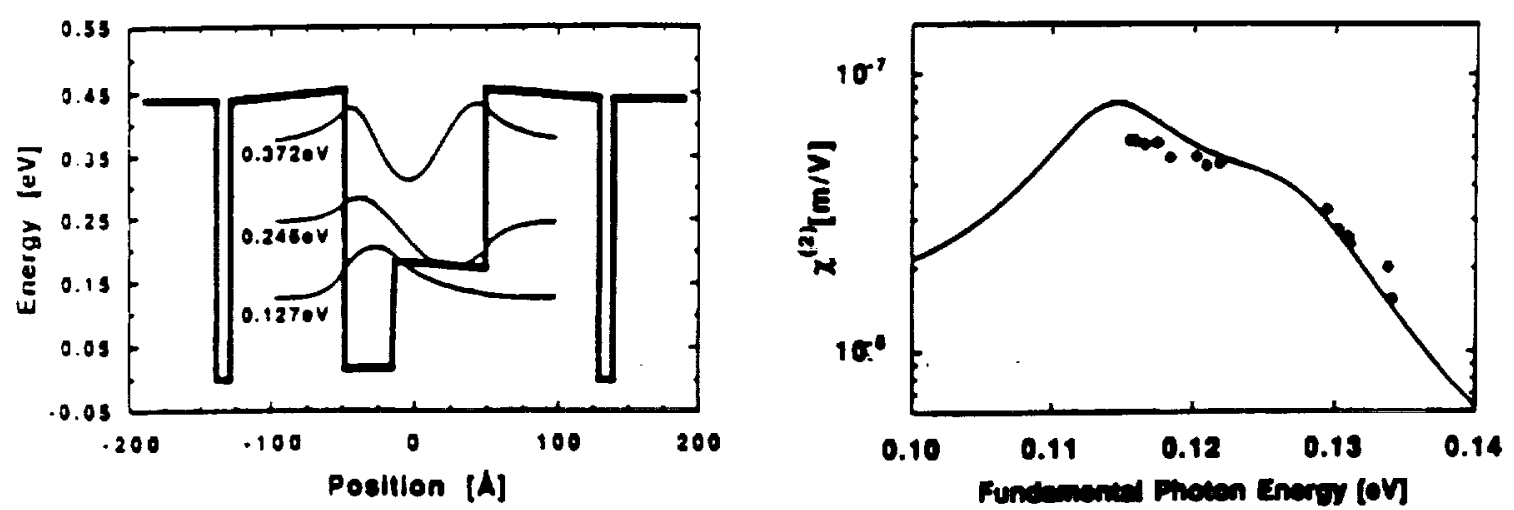

Figure 4 a) Diagram of the compositionally asymetric quantum well structure and the predicted subband wave functions. b) Measured (diamonds) and predicted (solid curve) nonlinear susceptibility near the carbon dioxide laser bands.

\section{CONCLUSION}

The advances in nonlinear materials and laser sources has combined to allow rapid progress in nonlinear devices. The early methodology of identifying a bulk crystal, preparing it for a nonlinear interaction and then carrying out the interaction with a laser is no longer an adequate approach. 
Today, the synthesis of the nonlinear material, whether an oxide or a semiconductor, or even an organic or polymeric material, is coordinated with the nonlinear material atomic structure. Examples of such structures include ferroelectric domains, quantum wells, patterned growth or modification of the nonlinearity. The architecture of the device must then be integrated into the concept for the device to meet the expected performance goals. For example, quasiphasematching in bulk or in guided wave geometries must be evaluated along with possible resonant enhancement geometries to achieve efficient conversion. The synthesis, structure, and architecture choices are driven by the ultimate device applications. What is new is that all of these issues must be taken into account in an integrated fashion to optimize the the use of a nonlinear material.

Thirty years after the first demonstration of a nonlinear optical process, nonlinear optics is ready to take the step from the laboratory to widespread use. Perhaps the efficient doubling of a diode laser to generate blue light will be the first nonlinear optical device to be found in automobiles.

Acknowledgements: This work was supported in part by the Army Research Office, the Office of Naval Research, DARPA, the National Science Foundation, NASA and by Sony Corporation.

\section{REFERWNCES}

1. D. R. Scifres, R. D. Burnham, W. Streifer, Appl. Phys Lett. 33 (1978) 1015

2. R. L. Byer, Science 239 (1988) 742

3. T. Y. Fan and R. L. Byer, IEEE J. Quantum Electr. 24 (1988) 896

4. R. Newman, J. Appl. Phys. 34 (1963) 437

5. B. K. Zhou, T. J. Kane, G. J. Dixon and R. L. Byer Opt. Lett. 10 (1985) 62

6. T. J. Kane and R. L. Byer, Opt. Lett. 10 (1985) 65

7. A. C. Nilsson, E. K. Gustafson, R. L. Byer, IEEE Journ. Q. Electr. 25 (1989) 767

8. T. Day, E. K. Gustafson and R. L. Byer, Opt. Lett. 15 (1990) 221

9. T. Day, E. K. Gustafson and R. L. Byer, IEEE J. Q. Electr. (to be pub)

10. D. Shoemaker, A. Brillet, C. N. Man, O. Cregut, Opt. Lett. 14 (1989) 609

11. P. Fritschel, A. Jeffries, T. J. Kane, Opt. Lett. 14 (1989) 993

12. R. Adler, Proc. IRE 34 (1946) 351

13. C. D. Nabors, A. D. Farinas, T. Day, S. T. Yang, E. K. Gustafson. R. L. Byer Opt. Lett. 14 (1989) 1189

14 D. L. Sipes Appl. Phys. Lett. 47 (1985) 74

15. E. A. P. Cheng and T. J. Kane, Opt. Lett. 16 (1991) 478

16. S. C. Tidwell, J. F. Seamans, C. E. Hamilton, C. H. Muller, D. D. Lowenthal, Opt. Lett. 16 (1991) 584

17. J. M. Eggleston, T. J. Kane, K. Kuhn, J. Unternahrer, R. L. Byer, IEEE J. Quantum Electron. QE-20 (1984) 289

18. S. Basu and R. L. Byer, Applied Optics 29 (1990) 1765 
19. T. Y. Fan, G. J. Dixon, R. L. Byer, Opt. Lett. $11(1986) 788$

20. T. Baer, J. Opt. Soc. Am. B 3 (1986) 1175

21. M. Oka and S. Kubota, Opt. Lett. 13 (1988) 805

22. A. Ashkin, G. D. Boyd, J. M. Dziedzic, IEEE J. Q. Electr. QE-2 (1966) 109

23. W. J. Kozlovsky, C. D. Nabors, R. L. Byer, IEEE J. Q. Electr. 24 (1988) 913

24 D. C. Gerstenberger, G. E. Tye, R. W. Wallace, Opt. Lett. 16 (1991) 992

25. W. K. Kozlovsky, W. Lenth, E. E. Latta, A. Moser, G. B. Bona, Appl. Phys. Lett. 56 (1990) 2291

26. S. T. Yang, C. C. Pohalski, E. K. Guztafson and R. L. Byer Opt. Lett. 16 (1991) 1493

27. D. H. Jundt, M. M. Fejer, R. L. Byer IEEE J. Q. Electr. 26 (1990) 135

28. D. H. Jundt, M. M. Fejer, R. L. Byer, Opt. Lett. (to be published)

29. J. A. Giordmain and R. C. Miller, Phys Rev. Lett. 14 (1965) 973

30 C. D. Nabors, R. C. Eckardt, W. J. Kozlovsky, R. L. Byer Opt. Lett. 14 (1989) 1134

31. C. D. Nabors, S. T. Yang, T. Day, R. L. Byer, J. Opt. Soc. Am. B 7 (1990) 815

32. R. C. Eckardt, C. D. Nabors, W. J. Kozlovsky, R. L. Byer, J. Opt. Soc. Am. B $8(1991) 646$

33. R. C. Eckardt, H. Masuda, Y. X. Fan, R. L. Byer, IEEE J. Quantum Electron. 26 (1990) 922

34. P. Bordiu, Crystal Technology (private communication)

35. R. Byer in Nonlinear Optics, ed by P. G. Harper and B. S. Wherrett, Academic Press, New York 1977 p47-160

36. Y. X. Fan, R. C. Eckardt, R. L. Byer, Appl. Phys. Lett. 53 (1988) 2014

37. Y. Wang, Z. Xu, D. Deng, W. Zheng, B. Wu, C. Chen, Appl. Phys. Lett. 59 (1991) 531

38. J. A. Armstrong, N. Bloembergen, J. Ducuing, P. S. Pershan, Phys. Rev. 127 (1962) 1918

39. N. Bloembergen U. S. Patent 3,384,433 May 21, 1968

40. G. A. Magel, M. M. Fejer, R. L. Byer, Appl. Phys. Lett. 56 (1990) 108

41. E. J. Lim, M. M. Fejer; R. L. Byer, Electron Lett. 25 (1989) 174

42. M. M. Fejer, J. L. Nightengale, G. A. Magel, R. L. Byer, Rev. Sci. Instrum. $55(1984) 1791$

43. D. H. Jundt, G. A. Magel, M. M. Fejer, R. L. Byer, Appl. Phy. Lett. (to be published)

44. M. M. Fejer, G. A. Magel, D. H. Jundt, R. L. Byer, Quasi-Phase-Matched Second Harmonic Generation: Tuning and Tolerances (submitted to J. Quantum. Electron.)

45. E. J. Lim, M. M. Fejer, R. L. Byer, Electronics Lett. 25 (1989) 731

46. R. L. Byer, M. M. Fejer, E. J. Lim U. S. Patent 5,036,220 July 30, 1991

47. Ya-lin Lu, L. Mao, Shi-de Cheng, Nai-ben Ming, Yu-tian Lu, Appl. Phys. Lett.59 (1991) 516

48. K. Mizuuchi, K. Yamamoto, T. Taniuchi, Appl. Phys. Lett. 58 (1991) 2732

49. C. J. van der Poel, J. D. Bierlein, J. B. Brown, S. Colak, Appl. Phys. Lett. 57 (1990) 2074

50. H. Ito, C. Takyu, H. Inaba, Electron. Lett. 27 (1991) 1221 
51. E. J. Lim, H. M. Hertz, M. L. Bortz, and M. M. Fejer, Appl. Phys. Lett. 59 28 October (1991)

52. E. J. Lim, S. Matsumoto, M. M. Fejer, Appl. Phys. Lett. 57 (1990) 2294

53. M. M. Fejer, Nonlinear Frequency Conversion in Periodically-Poled Ferroelectric Waveguides, (to be published)

54. L. C. West, S. J. Eglash, Appl. Phys. Lett. 46 (1985) 1156

55. M. M. Fejer, S. J. B. Yoo, and R. L. Byer, A. Harwit, J. S. Harris, Phys. Rev. Lett. 62 (1989) 1041

56. S. J. B. Yoo, M. M. Fejer, R. L. Byer, J. S. Harris, Appl. Phys. Lett. 58 (1991) 1724

57. R. Lodenkamper, M. M. Fejer, J. S. Harris, Jr. "Surface Emitting Second Harmonic Generation in a Vertical Resonator" (to be published) 